\title{
Perspectives of Application of New Modifications of Resonant Quasi-Optical Structures in EHF Equipment and Electronics
}

\author{
G.S. Vorobyov, M.V. Petrovsky, V.O. Zhurba, A.I. Ruban, \\ Sumy State University
}

O.I. Belous and A.I. Fisun

A. Usikov Institute of Radio Physics and Electronics, National Academy of Sciences of Ukraine 12, Academician Proskura St., Kharkiv 61085, Ukraine

\begin{abstract}
General properties of the "classic" open resonators without heterogeneities as the base devices while determining the particularities of new modifications of the millimeter-wave and sub-millimeter-wave band resonant structures are disclosed in the paper. The properties of open resonators with periodic metal heterogeneities, coupled open resonators as well as various modifications of resonant metal-dielectric structures are considered. The way for further investigation of new modifications of the resonant quasi-optical structures is determined from the point of view of optimization of their output parameters.
\end{abstract}

\section{INTRODUCTION}

Open resonators (OR) being the most important components of quite a number of devices [1-5] found wide application as the resonant systems in the equipment and electronics of millimeter and sub-millimeter (MSM) wave bands. In particular, a series of efficiently operating MSM-wave band measurement devices - resonant wave meters with plane and concave metal mirrors, reference oscillators, resonant systems of the devices used for investigation of properties of the substances $[1,6-8]$ - is developed on the basis of OR. Semi-spherical and sphero-cylindrical OR, upon one of the mirrors of which the reflective diffraction grating (DR) is positioned, are used at creation of various modifications of the diffraction radiation oscillators (DRO) [2,9-11], having as compared to the "classical" devices (BWT, klystron, magnetron) improved

ISSN 0040-2508 (C) 2007 Begell House, Inc. 
frequency stability and output power. On the basis of OR with corner-echelette mirrors there are created the efficient semiconductor oscillators on the basis of Gunn diodes and IMPATT diodes, quasi-optical oscillatory systems of which are realized based on the circuit with reactive reflecting and transmission resonators [12]. Open resonators found practical application as an oscillatory system in the relativistic electronics. Different modifications of oscillators and amplifiers are created on their basis [13-15].

At the same time, a wide implementation of MSM-waves into the most different spheres of science and technology is setting new requirements to the output parameters and functional capabilities of the devices within this bandwidth.

Modification of electrodynamic system of these devices in order to increase the throughput bandwidth and to improve the efficiency of transformation of the electron beam (EB) energy into the oscillation power is one of the ways of their further development. While solving these problems new approaches were required. They were suggested and realized in the form of various modifications of multi-coupling open electrodynamic systems like coupled OR [10,16,17], open waveguides (OW) [5,15,18-20], OR with dispersion elements [12], as well as the devices with metal-dielectric structures (MDS) [21-23], on the basis of which it was possible to realize the Cherenkov diffraction mechanisms of waves excitation [10]. Reasonability of the above solutions is substantiated in [24]. However, the absence of the systematic approach to investigation of the aforementioned devices is hampering their practical realization.

The objective of the present review is to perform the comparative analysis of electrodynamic properties of "classical" open resonators with new modifications of resonant quasi-optical structures and to develop the ways of their further development on the above basis.

Based on the objective set above, this review is briefly disclosing the basic properties of "classical" OR without heterogeneities, as the base devices, while determining the particularities of characteristics of new modifications of the MSM-waves resonant structures. The properties of OR with periodic metal heterogeneities related to OR and of various OR modifications with resonant metal-dielectric structures are considered.

\section{MAIN PROPERTIES OF CLASSICAL RESONANCE QUASI- OPTICAL STRUCTURES}

Two-mirror open resonators without heterogeneities can be referred to the resonant quasi-optical structures.

In the simplest case an open resonator consists of two plane infinitely thin disks positioned in parallel to each other so that their axes of symmetry form a 
match. This resonator is called the plane-parallel one and represents an analog of the Fabri-Perot interferometer as well-known in optics.

The plane-parallel resonators possess a number of valuable properties: they have sparse spectrum of resonance frequencies, homogeneous field along the resonator symmetry axis and the wavelength in the resonator is slightly different from the wavelength in the free space [1,4-6].

However, the complexity of their adjustment, comparatively large dimensions and insufficient separation of the oscillation modes upon the types of loss resulted in the fact that the resonators with the reflectors possessing the property of quadratic phase correction appear to be more perspective in the MSM-wave bands. The above type of resonators is called the confocal resonators, which consist of spherical mirrors. These resonators possess a larger resolution as compared to the plane-parallel ones. Besides, confocal resonators are less sensitive to misalignment. For the resonator with spherical reflectors a substantially less loss of power per one propagation is typical as compared to the open resonator having plane mirrors and the same aperture. Its significant advantage lies in large separation upon losses of the basic and the higher oscillation modes [1,3-5], which are usually designated as $T E M_{m n q}$ [25], where the indices $m, n=0,1,2, \ldots$ describe the transverse components of the oscillations and $q$ as the longitudinal index of oscillations is determining the number of half-waves, which could be laid upon the axis of the OR. For the resonator with spherical mirrors the resonance distances or the resonance wavelengths of the oscillation modes must satisfy the following condition:

$$
\frac{2 H}{\lambda}=q+\frac{1}{\pi}(m+2 n+1) \arccos \sqrt{g_{1} g_{2}},
$$

where $H$ is the distance between the mirrors; $\lambda$ is the wavelength in the open resonator; $g_{1}=1-\frac{H}{R_{1}} ; g_{2}=1-\frac{H}{R_{2}} ; R_{1}, R_{2}$ are the curvature radii of the mirrors.

Limiting of the OR apertures results in occurrence of losses related to the wave emission into the free space and is poorly influencing upon the distribution of fields in the open resonator. Hence it follows that in order for the losses to be small the field has to be concentrated around the center of the mirror. This, in its turn, implies restrictions upon the selection of correlations between the curvature radii of the mirrors and the distance between them. To obtain the resonators, the field of which drops rather quickly at increasing of the radial coordinate, the distance between the mirrors must be selected within the following intervals:

$$
0<g_{1} g_{2}<1
$$


The latest expression is called the condition of "stability" of the resonator with quadratic correction [1], and $g_{1} g_{2}$ are the some geometrical parameters.

The particularities of the oscillations of different modes in the plane-parallel and in the spherical-mirror resonators are essentially different $[1,3,4]$. Distribution of the field between the plane plates is to a greater extent dependent upon the dimensions of the latter while field distribution upon the mirror with the spherical profile is mostly determined by the value of its curvature and the correlation $\frac{L}{R}$ of the distance between the mirrors, $L$ to the radius of their curvature, $R$.

The semi-spherical resonators including one plane and one spherical mirror have also got wide application in the MSM-wave devices. It is known that the basic oscillations within a semi-spherical OR are represented by the azimuthally homogeneous oscillations $T E M_{m 0 q}[1,3]$. If the field spot upon the plane mirror is substantially less than its diameter then the semi-spherical resonators can be substituted with a large degree of accuracy by the equivalent spheroid resonators where the distance between the mirrors is doubled. At that, the distribution of amplitudes in both types of resonators is practically identical. The Q-factor of oscillation in the semi-spherical OR depends upon the diffraction losses at the edges of the plane and the spherical mirror, Ohm losses at the same mirrors, the communication losses and the losses related to attenuation in the medium.

OR with the mirrors performed in the form of two-sided reflectors [1,4] can also be efficiently used as the oscillatory system. The resonator with two-sided reflectors is characterized by very small values of diffraction loss and is comparatively little sensitive to skewing of the reflectors. Its manufacturing in the millimeter-wave band is easier as compared to the confocal resonator.

Unlike the spherical reflector, which is characterized by a linear value - the curvature radius, two-sided reflector characterized by the angle between its sides $\alpha$. It is for that reason that the conditions of the geometric stability are substantially different in both cases. Each resonator with two-sided reflectors is stable under the condition that the side width is rather large.

The resonance wavelengths of the oscillation modes for the resonator with two-sided reflectors must satisfy the following correlation:

$$
\lambda=\frac{2 L}{q}\left[1-\frac{1}{2}\left(\frac{3 \alpha(2 m+1)}{4 q}\right)\right]^{\frac{2}{3}}
$$

As can be seen from formula, the made degeneracy is not observed in this resonator type with the exception of random one. 
The adjustment of the resonator with two-sided reflectors is not very much influenced upon by small turns of reflectors around their top. More serious losses can be caused by insufficient parallelism of the reflectors edges. Right angle positioning of the mirrors with respect of each other allows to eliminate the above losses. In this case, each of the two planes of symmetry of the resonator is representing in itself a set of resonators with two-sided and plane reflectors. This resonator has the properties similar to those of a semi-confocal resonator and is less critical with respect to an adjustment.

\section{RESONATORS WITH PERIODIC METAL HETEROGENEITIES}

In order to use the described above structures in EHF electronics it is suggested to introduce a periodic heterogeneity of the diffraction grating type (Fig. 1) into the semi-spherical OR. Such an electrodynamic system is used in diffraction radiation oscillators $[9,10]$.

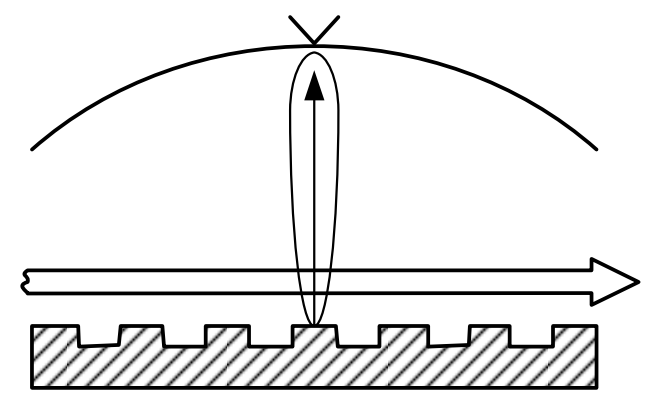

FIGURE 1. Semispherical OR with diffraction grating

The DRO operation principle is based on the diffraction radiation effect, which is excited by the electron beam propagating in the vicinity of the diffraction grating positioned in OR [9,10,26-33]. At that, while interacting with the diffracted upon the grating incident field, electromagnetic waves gain and oscillation modes are realized. Therefore, the DRO output characteristics are essentially determined by the properties of the applied OR. The periodic structure in OR of DRO is substantially varying the characteristics of the described above classical resonant quasi-optical structures. While performing the plane mirror in the form of the reflective DG [29-33], the total loss is essentially increased as the result of what the Q-factor for such a system is decreasing by almost four times. Decreasing of the Q-factor occurs as the result of additional losses, which are originated at power leakage for oscillation of the 
waveguide waves propagating along the grooves to the edges of the mirror where the reflection coefficient is not equal to one.

To eliminate this phenomenon we suggested the semi-spherical OR, in which only the central part of the plane mirror surface was covered with the diffraction grating $[9,10]$. This resonators has a rarefied spectrum of oscillations, the oscillation loss depends upon the parameters and the width of the grating. By varying the width of the grating we could essentially vary not only the number of the oscillation modes excited in OR but also control the distances over which the higher orders of oscillations could occur. Losses in OR are essentially dependent upon the correlation between the period of the grating and the lengths of the operation wave. After variation of the groove depths of the reflective grating the maximal Q-factor of the oscillations could be varied by several times.

In the semi-spherical OR with local DR $T E M_{20 q}$ is the fundamental mode.

The investigations results shown in $[9,10]$ proved that the excitation due to the comb filter is insignificant in such a system if the minimum of the field distribution is above the boundary between the grating and the mirror. This occurs at the DR width, which is larger than or equal to the width of the main lobe of the $T E M_{20 q}$ oscillation field.

Corner-echelette OR found wide applications at realization of the semiconductor sources of MSM waves. On the basis of the above electrodynamic systems the papers [34,35] suggest modifications of quasioptical solid-state pump oscillators with spherical corner-echelette OR, which are structurally realized upon the circuits with reactively reflecting and transmission-type resonators. As shown in [12] the oscillatory system of the corner-echelette OR possesses a number of particularities: the degree of sparseness of the corner-echelette OR spectrum is a bit less than of the spectrum of the OR with plane echelette mirror; however, the spectrum has the oscillation modes with an abnormally high Q-factor, which are classified as the quasi-basic modes of oscillations; the field of the quasi-basic oscillation modes is drawn to the OR axis and the density of their power is larger than for the basic ones and the rest of the oscillation modes; in the vicinity of the corner-echelette mirror the field structure undergoes a transformation and near the OR top it is similar to the structure of the field within a rectangular waveguide; corner-echelette mirror is the multi-step impedance transformer.

Variety of the relativistic electronics devices stipulated the necessity to develop special open electrodynamic resonant systems possessing enhanced electric resistance and efficient selection of oscillation modes. These resonant systems include the ring resonator represented by a set of mirrors positioned in the way that the beam being reflected from the resonant mirrors would be 'locked' upon itself. Separation of the optic beams of the direct and the reflected signals is the specific feature of such a resonator. Actually, the running waves 
mode is realized within the volume of the resonator. Besides, there occur at least two additional optical branches, through which other devices could be connected. The above particularity was used in one of options of the free electron laser (FEL) with stepwise frequency increasing. Application of Bragg resonators is also perspective within the MSM-wave band. Structurally, they consist of Fabri-Perot resonators, the mirrors of which form up serrated or wavelike mirror surfaces. These resonators are mosty applied in the FEL structures $[14,36,37]$. Being distinguished by its multi-functionality the Bragg resonator is a multi-frequency system. Moreover, for the pump wave propagated along the resonator axis the system of mirrors is also a highly selective decelerating structure.

\section{COUPLED OPEN RESONATORS}

In the papers $[10,16,17,29,38,39]$ there are suggested and partially investigated the diffraction electronics devices upon coupled OR, which, as compared to the single resonator OR, possess a number of advantages: having larger range of electronic frequency readjustment; capable of being efficiently used as the power amplifiers and frequency multipliers. OR coupling in such devices can be realized either by means of the field diffracted at the edges of the mirrors using in-series positioning of the resonators (Fig. 3(a)) or by means of the field diffracted upon the ribbon-type gratings using in-parallel connection of OR (Fig.3(b)) with respect of the axis of the distributed oscillation source. EB is such a source in the electronic devices. And in the case of experimental modeling of the processes of excitation of diffraction radiation (DR) it is the surface wave of the single-mode planar dielectric waveguide (DW).

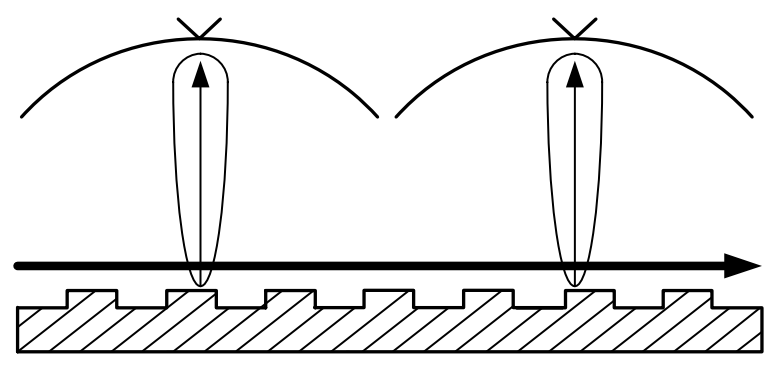

a)

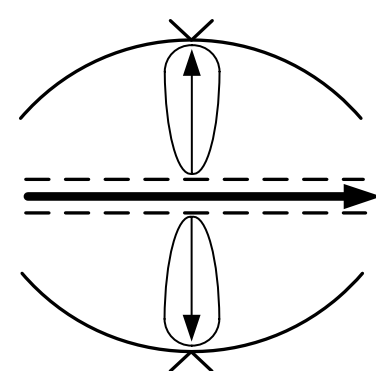

b)

FIGURE 2. Electrodynamic systems based on coupled OR: a) - in-series connection of OR; b) - in-parallel connection of OR 
The system of serially coupled OR (Fig. 2(a)) is, in the simplest case, represented by two semi-spherical resonators with the common plane mirror performed as the reflective DG. In the case with the system of in parallel coupled OR (Fig. 2(b)) ribbon-type diffraction gratings are positioned between the spherical mirrors.

Simulation of wave processes in the coupled OR with the oscillation source in the form of a planar dielectric waveguide is performed in [16,17,39]. In order to determine the particularities of the electrodynamic systems shown in Fig. 2, there were investigated the resonant characteristics of oscillations and the resonance frequencies spectra of the coupled OR, which characterized the probability of excitation of a limited number of $T E M_{m n q}$ oscillation modes in the given resonant systems. At that, the measurements of similar characteristics for the single semi-spherical and spheroid OR used to be the basic ones.

It is demonstrated that the throughput bandwidth of the coupled OR (Fig. 2(a)) is increasing by almost two times as compared to the base resonator and amounts to the value of $\Delta f \approx 250 \mathrm{MHz}$ within the $f=45 \div 47 \mathrm{GHz}$ frequency bandwidth. At that, the resonance curves of the coupled OR are witnessing for realization of the efficient coupling using the fields diffracted at the periphery of the mirrors while performing adjustment of the resonator to the close-range frequencies.

The results of investigation of the system (Fig. 2(b)) prove the fact that for the comparable values of the distances between the mirrors the maximal throughput bandwidth within a resonant system can be attained only at organization of the optimal coupling of two OR via the ribbon-type DG: the throughput bandwidth of the system at the in-parallel connection of OR is by almost 5 times larger than at the in-series connection of OR. In this case the Qfactor of the oscillations of the coupled OR is of the same order as that for the single OR. This effect is confirmed by the investigation results with respect of the coupled resonators as shown in the paper [40].

Therefore, it can be seen that the coupling between two resonators via the ribbon-type gratings has a perspective in terms of development of the diffraction grating devices, if it is required to broaden the bandwidth of the device or to decrease its dimensions along the electron beam axis. The latter property is simplifying the focusing of the electron beam.

The aforementioned resonant systems apply mostly the quasi-optical OR where the dimensions of their mirrors upon the curvature radius amount to $R \approx 20 \div 30 \lambda$, and upon the aperture - to $7 \div 10 \lambda$, which is restricting the practical opportunities of their use in several types of radio engineering equipment. These dimensions can be substantially diminished by means of using small-dimension OR with short-focus mirrors [10,41].

The spectral characteristics and distribution of internal resonant fields for the short-focus OR indicate at excitation of axially symmetrical oscillations, which 
form a qualitative match in terms of their structure with the fields formed by the long-focus reflectors. In [10] it is experimentally determined that the resonant oscillations are present in the spectrum of the short-focus OR at the relative distances between the mirrors of $\frac{L}{R}>1$. The Q-factor of these resonances is not high ( $Q \approx 300 \div 500$ ) due to large-value diffraction losses.

In the short-focus semi-spherical OR with locally positioned upon the plane mirror diffraction grating, unlike in the long-focus OR, the fundamental mode $T E M_{00 q}$ is present within the entire range of distances that indicates upon the perspectives of their application in EHF electronics.

The two-stage DRO [10] is realized on the basis of the system shown in Fig. 2(a) within the frequency bandwidth of $f=43 \div 98 \mathrm{GHz}$. This device is formed by two short-focus spherical mirrors and the common cylindrical mirror, along the longitudinal axis of which the diffraction grating is applied. The electron beam is formed by the diode gun and accompanied by the magnetic field it is propagated along the DG exciting oscillations within the system of coupled OR. In the event of a weak coupling between the ORs the device operates like a multifrequency oscillator at partial frequencies; under availability of the optimal coupling - like a broadband DRO upon the coupled OR, the bandwidth of the electron frequency readjustment is increased by more than 1.5 times as compared to the single-stage DRO that is confirmed by the results of investigations $[16,17]$.

In $[29,38]$ it is suggested the DRO upon the in-parallel coupled OR, the electrodynamic system of which is shown as a diagram in Fig. 2(b). It consists of two semi-spherical OR coupled via the slots of two identical ribbon-type DG, between which the EB is propagating. If the EB current is substantially larger than the starter current, then such a DRO is operating like an oscillator. If the signal from the external source of microwave oscillations is supplied to the input of one of the resonators and the condition of self-excitation is not fulfilled the device is operating like an amplifier [42]. Besides, due to availability of two coupled OR this device is capable of performing the functions of a frequency multiplier $[10,38]$.

In [16] it is described the system of coupled resonators in the form of an OR and the volume resonator with moving short-circuiting piston positioned at the opposite side from the coupling slot. With the help of mechanical readjustment of the volume resonator and at the fixed value of the distance between the mirrors of OR the oscillation frequency can be smoothly varied within the rather broadband limits and with the drop of the output power level not exceeding $3 \mathrm{~dB}$. This system of coupled resonators possesses improved vibration resistance properties as compared to the mechanical re-adjustment of the frequency by the OR mirrors. 


\section{METAL-DIELECTRIC RESONANT QUASI-OPTICAL STRUCTURES}

Multivariable coupled systems performed in the form of OR and OW, within the volumes of which the MDS is positioned, allow us to realize different modes of energy transformation depending upon the parameters of the electrodynamic system $[23,43-46]$.

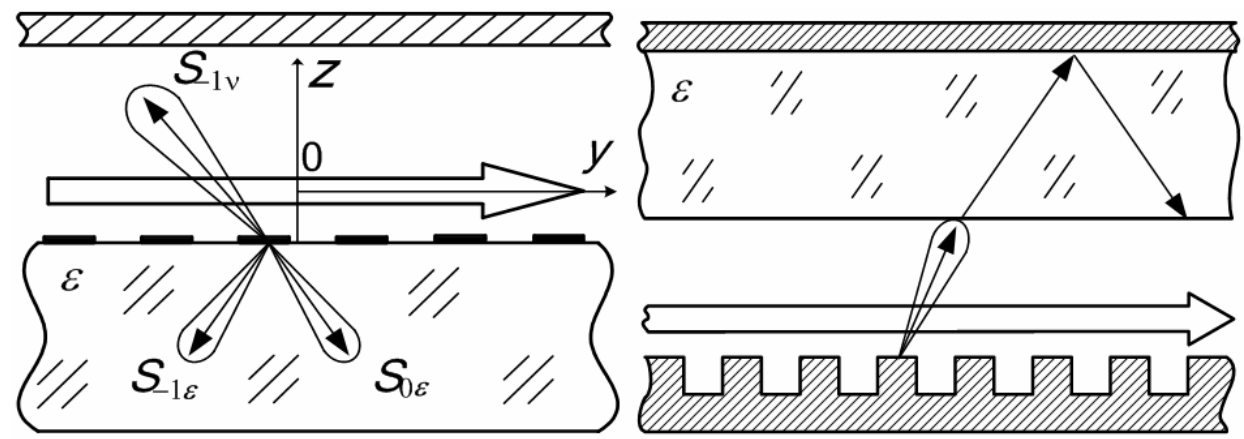

a)

b)

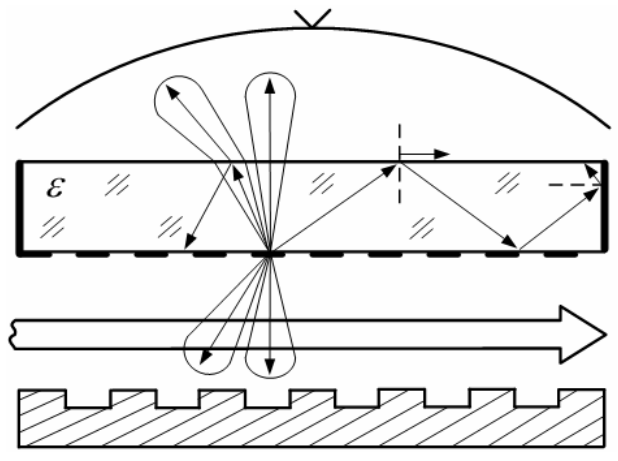

c)

FIGURE 3. Resonance quasi-optical structures with MDS

The simplest electrodynamic system of OR with MDS is shown as a diagram in Fig. 3(a). It is formed by the metal screen and the dielectric layer with the permeability $\varepsilon$, at the lateral side of which the ribbon-type diffraction grating [22] is applied. The distributed oscillation source, which is capable depending upon the system parameters of exciting various spatial harmonics of the Cherenkov diffraction radiation (ChDR) with the numbers $n=0, \pm 1, \pm 2 \ldots$ and the power density $S_{n}[10,22]$, is positioned along the grating. In particular, 
Fig. 3(a) demonstrates the option of excitation of the Cherenkov $\left(S_{0 \varepsilon}\right)$ and the minus first diffraction $\left(S_{-1 \varepsilon}\right)$ harmonics of oscillation into the dielectric, as well as of the minus first diffraction harmonic of oscillation $\left(S_{-1 v}\right)$ into the vacuum, which can be reflected from the metal screen and be supplied to the metal dielectric channel. Numerical and experimental methods for simulation of different modes of excitation of ChDR [47-50] allowing to determine the quantitative correlations of power densities of the spatial harmonics of oscillation and to optimize the parameters of the electrodynamic system in compliance with the set problem are developed for such a system.

The system of the open waveguide with MDS represented in Fig. 3(b) is perspective for regenerative and broadband amplification of electromagnetic waves upon the spatial harmonics of diffraction radiation (the Smith-Purcell oscillation) at propagation of the non-relativistic EB along the periodic structure. The linear self-matching theory of electromagnetic waves amplification based on the Smith-Purcell effect and considering the influence of the dielectric layer and the electron beam thickness upon the conditions for excitation of oscillations in an open waveguide [23,51-54] is developed for this system. It is determined that by varying of the electrodynamic parameters of $\mathrm{OW}$ it is possible to realize different modes of excitation of oscillations - DR oscillation mode upon the normal (regenerative mode), the mode of traveling volume wave and the surface waves mode - (BWT, TWT).

The natural transition from the simplest resonant structure systems (Figs. 3(a) and 3(b)) to more complicated ones is the open resonator with MDS (Fig. 3(c)), which is formed by, for instance, the spherical mirror with power output and the plane mirror with reflecting diffraction grating [22]. MDS performed as the dielectric resonator is positioned between the OR mirrors. Such an electrodynamic system forms the basis at creation of Cherenkov diffraction oscillators. Figure 3(c) demonstrates the possible modes of excitation of ChDR by a distributed oscillation source positioned in the vicinity of the boundary between the dielectric prism and the ribbon-type DG. A more detailed description of the wave modeling of ChDR in spatially limited MDS is provided in [55].

It is determined that introduction of the above described MDS (Fig. 3(c)) into the open resonator results in qualitatively new electrodynamic properties of such a system [22]: at variation of the MDS parameters it is possible to realize the modes of power attenuation in OR, to increase the amplitude and the value of Q-factor of oscillations and to improve the selection of oscillations. The detected properties of such a system are finding their explanation within the framework of the physical model of ChDR for MDS with finite length [55] and the known concept of representation of the resonant field in the form of an angular spectrum of the plane waves [56-58]. Subsequent investigations of the above systems [59-62] allowed to suggest and substantiate the specific circuits 
of the diffraction electronics devices with spatially developed resonant structures - the Cherenkov diffraction oscillator [22,60,61], Cherenkov BWT [22,60]. In particular, Fig. 4 presents the generalized diagram of execution of the Cherenkov BWT and the Cherenkov diffraction oscillator.

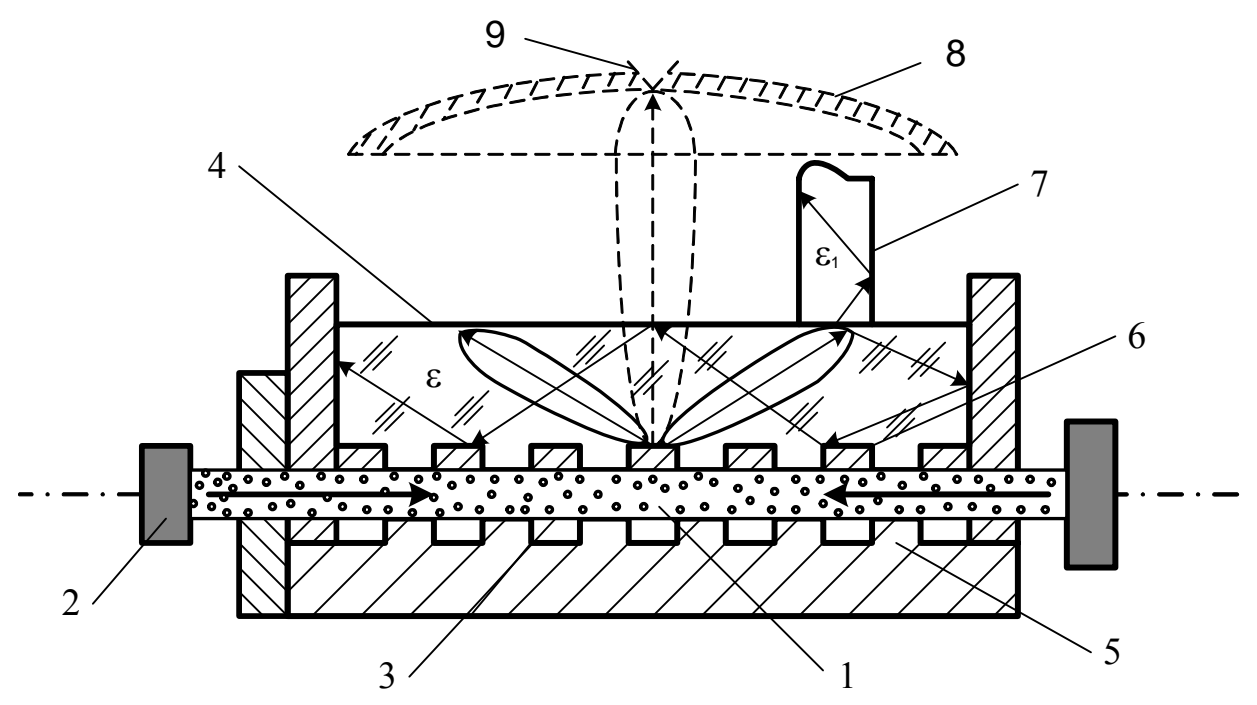

FIGURE 4. Generalized diagram of execution of Cherenkov BWT and Cherenkov diffraction oscillator

In the event of execution of BWT the electron beam 1 is generated by the gun 2 and transmitted via the channel 3 formed up by adjacent surfaces of the resonator 4 and the decelerating system 5 . The electrons are interacting with the field of the surface slow wave of the decelerating system 5 and are modulated upon the density of the charge. Simultaneously, at the electrons velocity exceeding the phase velocity of the wave in the dielectric there occurs $\mathrm{ChR}$, which is directed to the dielectric. The oscillation having the configuration of the field providing for the feedback (arrowed solid lines) is excited in the resonator 4. While forming a match of the frequency with one of the eigen frequencies of the resonator the oscillations are excited therein and there occurs an efficient sampling of the power via the ribbon-type grating 6 from the EB modulated upon the slow wave. The output of the power accumulated in the resonator 4 is performed via the waveguide 7 with $\varepsilon_{1}>\varepsilon$. The synchronous mode established between the groups of electrons and the wave in the dielectric is secured by the relevant selection of the parameters $\varepsilon$ and the accelerating voltage of $\mathrm{EB} U_{0}$. 
Similar electron optics is used for excitation of the Cherenkov diffraction radiation. The retardating system (diffraction grating) 5 is positioned in the central part of the fixed mirror of OR. The moving mirror 8 possessing the coupling slot 9 is used for providing the power output. Inlike the BWT, the periods and the filling coefficients of the gratings 5, 6 are determined upon the conditions of efficient excitation of DR upon the normal with respect of the axis of the diode gun 2 (dotted oscillation pattern in Fig. 4) and the optimal power density of $\mathrm{ChR}$ within the dielectric resonator 4 . The aforementioned devices can also be performed in other options, e.g. on the basis of the elements of a cylindrical shape using the axially symmetrical beams [63].

\section{MULTI-MIRROR OPEN RESONATORS WITH ECHELETTES}

It has already been mentioned that the electronics of millimeter-wave and submillimeter-wave bands applies open resonators with the elements, upon which there occurs interaction between the electron beam or a solid non-linear element and the fields in OR, and which serve as a matching link. The classical open resonant systems consisting of two mirrors are used mostly in the devices of quantum electronics where the homogeneous distributed easant is applied.

The paper [64] is investigating the resonators with the mirrors consisting of two echelettes. These resonators can be referred to the class with an additional restriction of the resonant space [65] because the diffraction coupling with the external medium is significantly decreased, unlike in the classical OR. The model [66] was suggested for the analysis of the oscillatory processes in OR of the similar type. Its basis is formed by the ideas allowing to interpret the OR eigen oscillations as the "locked-up" ones within the inner volume of the waveguide section, by which the OR is simulated [67]. The waveguide model of OR suggested by L. A. Weinstein has been modified for the case of excitation of OR by the external source. It allowed to solve the heterogeneous problem of excitation of oscillations in OR and to bring the computer experiment as maximally close as possible to the real (in situ) one. Methodically, solving of this problem is provided using the method of the generalized matrix of scattering. It has been detected that several types of oscillations, the Q-factor of which is exceeding the value of the Q-factor of the basic types of classical OR [68] are excited in the corner-echelette OR.

It is known that DRO (the orotron) possesses high degree of oscillation coherence as the result of high-Q value of the oscillatory system. This is to a full extent referred to the medium and low power devices. With the increase of the oscillated power the current has to be increased. As the result of that in the orotrons with the oscillatory system having weak selection properties (spherocylindrical OR), the closest oscillation zones are overlapped that impedes attaining of the maximum of the output power within the electron zone of re- 
adjustment. This problem can be solved only using the additional spectrum sparseness of the OR under the condition of high-Q value of the operation mode of the oscillations.

Using of oscillatory systems with additional dispersion elements within the powerful single-frequency orotrons is one of the way-outs from this situation. The paper [69] investigated the model of DRO (orotron) with the cornerechelette mirror [64], the other mirror was a spherical one. The slot-like power output is positioned therein. The spherical corner-echelette OR (SCE OR) is executed not only upon the $H$-polarized but also upon the $E$-polarized oscillations. The latter phenomenon allows to position the retardating comb grating in the top of the corner-echelette mirror and let the electron beam get propagated over the grating. Figure 5 represents the cross-section of the orotron model along the axis of the electron beam. The plane mirror contains the comb slowing grating 1 and the electron gun 2 .

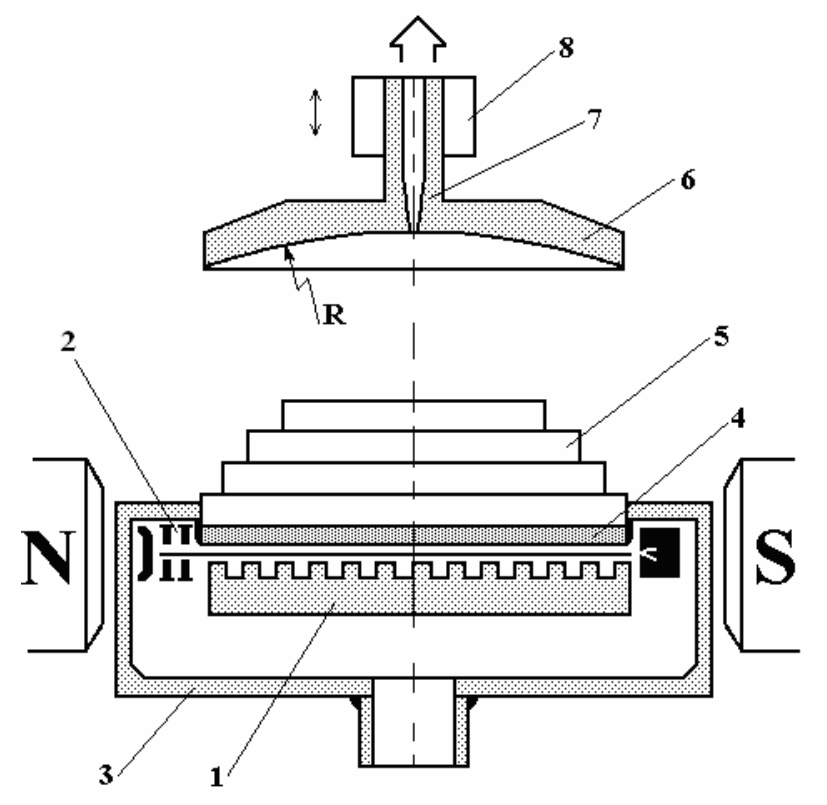

FIGURE 5. Cross-section of the orotron model with vacuum section. The oscillatory system is under the atmospheric pressure.

This part of the oscillator along with the electron beam is located in the vacuum chamber 3 . The transparent for the oscillation screen 4 is dividing the resonator into two parts. The other part of the SCE OR is subject to the atmospheric pressure. The corner-echelette mirror 5 is positioned upon the transparent screen 4 . The spherical mirror 6 having the output slot 7, is fixed 
within the travel gear 8 and possesses a power output. This structure of the model allowed to perform the comparative testing of the orotron with the planespherical OR and the orotron, within which the corner-echelette mirror has been installed; in so doing other parameters of the resonator as well as of the electron beam is remained unchanged.

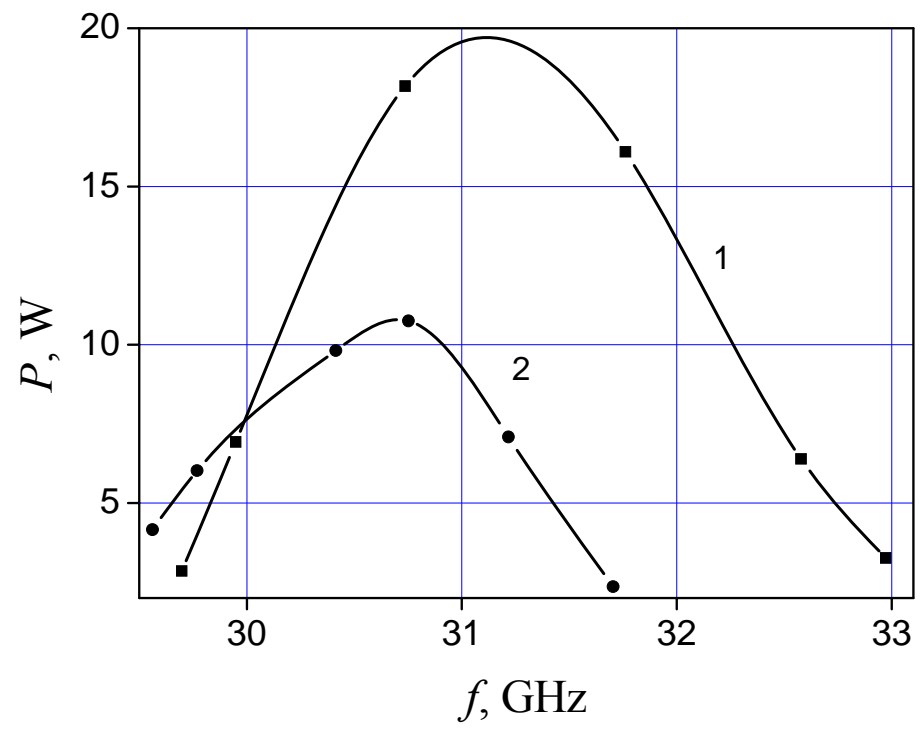

FIGURE 6. Frequency dependence of orotron oscillation upon the mechanical re-adjustment. 1 - orotron with SCEOR, 2 - orotron with plane-spherical OR

Figure 6 demonstrates the re-adjustment characteristics at the mechanical readjustment of the SCE OR length. For the purpose of comparison this paper provides for characteristics of the orotron with a traditional resonator, within which the simultaneous excitation of the basic and the higher modes is observed upon several correlations of $L / R$. For example, at $L / R=0.73 \div 0.75$ the traditional OR is excited simultaneously upon $T E M_{007}$ and $T E M_{017}$. At the same time along the large interval of variations of $L / R$ there were observed only fundamental (or quasi-fundamental) oscillation modes with various longitudinal numbers. This is explained by a good match of the beam with the oscillatory system as well as by the high-value Q-factor of the basic modes, at excitation of which the field is shrinking (i.e, concentrated in the vicinity of the resonator axis). The diffraction loss is, at that, becoming minimal. At application of SCE OR the generated power is increasing by approximately 1.5 to 1.7 times at the same value of the current. 


\section{METHODS OF EXPERIMENTAL INVESTIGATION AND CALCULATION OF PARAMETERS OF RESONANCE QUASI- OPTICAL STRUCTURES}

The possibility of efficient application of various modifications of OR in the MSM-wave band electronics and equipment is related to consideration of influence of their properties upon the electrodynamic characteristics of the devices. Therefore, such investigations are an independent problem including, as a rule, the following stages - determining of the spectrum of oscillations, the value of the transmission coefficient upon the power, Q-factor, amplitude distribution of the field between the mirrors. The above characteristics for real systems can be studied mostly experimentally because the theory of OR with various kinds of metal and metal-dielectric heterogeneities is represented as a complicated problem of electrodynamics, at solving of which you cannot receive specific answers with respect of realization of new modifications of the devices with spatially developed periodic structures.

In practice, measurement of the transmission coefficient of the resonator is performed by switching OR for transmission when the excitation of the system is executed from the part of one of the mirrors $[1,6,7,64]$. By moving the mirrors of the system along the axis of the OR or by varying the frequency of the exciting oscillations within the set bandwidth there could be adjusted the power of the signal, which would be proportional to the transmission coefficient of the resonator upon power.

Variation of the open resonator spectrum is reduced either to determining of the resonance frequencies at the fixed distances between the mirrors or to determining of the resonance distances between the mirrors of the resonator at the fixed frequencies of the oscillation source $[64,65]$.

Another important characteristic of the oscillatory system is its Figure of merit. In the general case the Q-factor is determined upon the formula $[1,7,28]$ :

$$
Q=\frac{2 \pi L}{\lambda \delta}
$$

From the correlation (1) it is evident that the Q-factor of OR is directly proportional to the distance between the mirrors, i.e., the resonant volume, and adversely proportional to the aggregate value of loss $\delta$. Depending upon what is meant by $\delta$ - the losses of loaded or not loaded resonator - the eigen Q-factor or the loaded Q-factor are determined from this correlation. The Q-factor of the loaded resonator can be determined upon its frequency characteristic. Having taken the dependence of the transmission coefficient on the power upon the frequency and after measuring the width of the resonant curve $\Delta f$ at the level of 0.5 , the resonator Q-factor is found from the correlation: 


$$
Q_{i}=\frac{f_{0}}{\Delta f}
$$

where $f_{0}$ is the frequency matching with the power maximum of the resonant curve.

The schematic solution allowing to diminish the measurement error or the Q-factor of transmission resonators by several times is realized using the reference resonator for the system of automated adjustment of the oscillator frequency. At that, smooth re-adjustment of the oscillator frequency is performed by moving one of the mirrors of the reference OR with synchronous recording of the transmission coefficient of the OR under investigation $[1,7]$.

Alongside with the above the significant attention is paid in the MSM-wave band devices to investigation of the structure of the electromagnetic field distribution. The configuration of this field plays an important part in the systems realized on the basis of OR.

For this purpose in $[2,9,28,70]$ there are developed the methods of reactive probing permitting to perform visualization of the electromagnetic fields in OR as well as to measure the relative amplitudes of distribution of the fields in OR. The essence of the method lies in the fact that the probe is entered into the resonant volume as a reflective or absorbing body, the dimension of which is selected depending upon the value of the amplitude of the measured field and more than often it is less than the wavelength of the resonant oscillations - the method of the sample body [9]. The sample body is imparted a trajectory of the motion upon the surface within the resonant volume of OR. The structure of the field is judged upon the characteristics of the field scattered by the probe or upon the parameters of the disturbed system - variation of the Q-factor or, what is equivalent, of the transmission coefficient of OR. Using the dependence of the transmission coefficient of the resonator upon the amplitude of the field in the place where the probe is positioned, the amplitude distribution of the OR field is measured.

In $[9,10]$ it is demonstrated that the method of experimental modeling, at which the oscillation of the electron wave of the spatial charge current of EB is simulated by means of the oscillation of the surface wave of the planar dielectric waveguide positioned in the vicinity of the diffraction grating, is an efficient method for investigation of new modifications of electrodynamic systems of the diffraction electronics devices. At uniform and straight line motion of the electron beam its eigen field has the form of a plane wave. Similar wave can be formed by a plane dielectric waveguide. A part of the power propagated along the waveguide is concentrated within the external domain with respect of the waveguide in the form of a slow waves surface field stipulating its diffraction upon the elements of the periodic structure. This allows to simulate the diffraction radiation effect with the help of the waveguide fields only. Resulting 
from the above the power of the slow waves is transformed into the power of the fast spatial harmonics, which is radiated into the environment.

Theoretical substantiation of this method under the condition of excitation of the spatial harmonics of oscillation upon the MDS is provided in [10]. It is determined, that the surface wave of DW may be transformed into a volume wave similar that electronic beam one. It is possible the different mode transformations. The most typical of them can be clearly analyzed by means of building Brillouin diagrams for different values of dielectric permeability of the medium [71]. Thus, by varying the parameters of the dielectric waveguide, the grating and the dielectric medium the models of Cherenkov, diffraction or Cherenkov diffraction radiations can be realized.

On the basis of this approach experimental modeling of the diffraction radiation effect allows to determine the number of spatial harmonics, direction of their maximal oscillation, dependence of the power upon the wavelength, polarization and the target distance of the dielectric waveguide with respect of the diffraction grating $[9,10]$.

In addition to the aforementioned experimental methods of investigation of the resonant quasi-optical structures there exists a vast class of theoretical calculation methods based on both the analytical and the numerical solution of the problems, which allow to qualitatively and quantitatively predict the physical processes occurring in the given system under investigation.

In particular, for the above described classical resonant quasi-optical structures Fox and Lee solved the problem of determining of the fields distribution, the resonant frequencies spectrum and oscillation losses, stipulating alongside with the Joule losses the unloaded Q-factor of the resonator [1], which has been reduced to solving of the homogeneous integral Fredholm equation of the second kind. Fox and Lee chose the iteration methodology in order to solve this equation.

In [72] Cullen developed the scalar and the vector theories for both the base OR and the OR with the heterogeneity in the form of a dielectric plate positioned between the mirrors of the resonator. The obtained in the analytical representation formulas link together the parameters of OR and the dielectric with the oscillation frequencies of the resonator that allows considering the influence of the dielectric layer upon the oscillation spectrum of the base OR. Based on the vector theory where the full consideration of the diffraction effects is performed, the precise formula of the resonant frequency of the fundamental mode TEM $00 q$ is obtained for the base spheroid OR and for the OR with the dielectric layer there are obtained transcendental equations relating the frequencies of symmetrical and asymmetrical TEM 00 modes, allowing to consider their influence upon the oscillation spectrum of the base OR.

The analysis of excitation of OR with step-like deformation of the mirrors is developed in $[66,68]$. It is based upon the waveguide model of OR, generalized 
matrix of scattering and is performed with the help of the automated system of electrodynamic simulation $[73,74]$. Nowadays, due to the increased operation speed of PC the FDTD (Finite-Difference Time-Domain) method which is one of the basic methods for numerical solution of the problems of electrodynamics $[67,68]$ is finding still wider application. The FDTD method is a versatile one it could be successfully applied for practically any problem of electrodynamics requiring the numerical solution.

The frequency characteristics of the object under investigation can be obtained with the help of the discrete Fourier transformation or conditionally by means of setting of the quasi-harmonic source and execution of calculations up to attaining of the set mode. In addition to the simplicity of setting of the problem, the FDTD method possesses undisputable advantages with respect of simulation of the electrodynamic objects with heterogeneous, anisotropic and non-linear media having arbitrary shaped boundaries.

In its classical setting the FDTD method is based upon discretization of the Maxwell equations put down in the form of a differential space and time formulation [69].

\section{CONCLUSION}

The paper provides systematization of the investigation results of both classical open resonant structures forming the basis at development of new modifications of oscillatory systems of the MSM-wave band devices and equipment and more complicated electrodynamic systems with spatially developed periodic structures - coupled OR, open resonators and waveguides with MDS. It is demonstrated that the coupled OR possess wider frequency re-adjustment bandwidth while preserving high values of the Q-factor of oscillations. Multifunctional systems performed as OR and waveguides with MDS have qualitatively new properties by varying the MDS parameters there could be attained realization of the attenuation mode or increasing of the amplitude of oscillations and their selection. New modifications of the Cherenkov BWT and the Cherenkov diffraction oscillator are suggested on this basis.

The methods for experimental and theoretical investigations of the resonant quasi-optical structures like the sample body method, the method of waveguide simulation, vector theory and numerical calculation with the help of the finite differences method within the FDTD time domain are briefly analyzed. The whole complex of the above methods allows to efficiently conduct at the initial stage of the experimental research the analysis of waveguide processes in complicated resonant structures including dispersion elements like reflective and ribbon-type diffraction gratings as well as metal-dielectric structures. 


\section{REFERENCES}

1. Valitov, R.A., Dyubko, S.F., Kamyshan, V.V. et al., (1969), Submillimeter-wave equipment, Sov. Radio, Moscow: 480 p. (in Russian).

2. Shestopalov, V.P., (1985), Physical basis for millimeter- and submillimeter-wave equipment, Naukova Dumka, Kyiv, Vol.1:216p. (Open-type structures), Vol.2:256p. (Sources, element basis. - Radio systems) (in Russian).

3. Weinstein, L.A., (1966), Open resonators and open waveguides. Sov. Radio, Moscow: 475 p. (in Russian).

4. Katsenelenbaum, B.Z. and Shevchenko, V.V., (1966), Quasi-optics, Mir, Moscow:428 p. (in Russian).

5. Weinstein, L.A., (1995), Theory of diffraction. Microwave electronics, Radio i Svyaz, Moscow: 600 p. (in Russian).

6. Milovanov, O.S., and Sobenin, N.P., (1980), Microwave equipment. Atomizdat, Moscow: 464 p. (in Russian).

7. Valitov, R.A., and Makarenko, B.I., (1984), Measurements upon millimeter and submillimeter waves: Methods and equipment, Radio i Svyaz, Moscow: 296 p. (in Russian).

8. Lebedev, I.V., (1970), Microwave equipment and devices. Higher School, Moscow:440 p. (in Russian).

9. Shestopalov, V.P., (1976), Diffraction electronics. Kharkiv University Publishers, Kharkiv: 232 p. (in Russian).

10. Shestopalov, V.P., (1991), Diffraction radiation oscillators, Naukova Dumka, Kyiv:320 p. (in Russian).

11. Vavriv, D.M., and Tretyakov,O.A., (1989), Theory of resonant amplifiers with distributed O-type interaction. Naukova Dumka, Kyiv: 152 p. (in Russian).

12. Fisun, A.I., and Belous, O.I., (1998), Quasi-optical solid-state sources of oscillation - basics of creation, trends of development and perspectives of application, Foreign electronics. Achievements of present-day radio electronics.4:41-64.

13. Balakirev, VA., Karbushev, N.I., Ostrovsky, A.O., Tkach, Yu.V., (1993), Theory of Cherenkov amplifiers and oscillators based on relativistic interaction of oscillation bundles, Naukova Dumka, Kyiv: 192 p. (in Russian).

14. Ginzburg, N.S., Sergeyev, A.S., and Peskov, N.Yu., (1999), On possibilities of application of two-dimensional Bragg structures in FEL-based amplifier fed by ribbon-type electron beam, Letters to JTP.25(19):87-95 (in Russian).

15. Weinstein, L.A., and Solntsev, V.A., (1973), Lectures on microwave electronics, Sov. Radio, Moscow: 400 p. (in Russian).

16. Vorobyov, G.S., (2000), Electrodynamic properties of coupled quasi-optical open cavities in sources of millimeter radiation, Laser Physics.10(4):932-938.

17. Vorobyov, G.S., (2001), Experimental modeling of conditions of excitation of oscillations in electrodynamic system of coupled open resonators, Ukrainian Physical Journal. 4(10):1043-1046 (in Russian).

18. Vertiy, A.A., Vorobyov, G.S., and Ivanchenko, I.V., (1988), Experimental investigations of transformation of surface waves into volume ones in open waveguide, Procs. of Higher School. Radio Physics.31(6):1242-1254 (in Russian). 
19. Vorobyov, G.S., Ruban, A.I., and Shmatko, A.A., (1999), Linear theory of nonresonant EHF amplifier with distri-buted interaction based on Smith-Pursell effect, Procs. Higher School. Radio electronics. 42(6):67-70 (in Russian).

20. Vorobyov, G.S., Ruban, A.I., Kryvets, A.S., and Shmatko, A.A., (1999), Theoretical analysis of conditions for excitation of oscillations in amplifier with distributed interaction based on Smith-Pursell effect, Procs. Sumy State University. 1(12):28-33 (in Russian).

21. Vorobyov, G.S., Makeyev, O.S., Pushkaryov, K.A., and Tsvyk, A.I., (1996), Application of metal dielectric periodic structures in EHF electronics, Procs. Sumy State University. 1(5):17-22 (in Russian).

22. Vorobyov, G.S., Kryvets, A.S., Petrovsky, M.V., Ruban, A.I., Tsvyk, A.I., (2003), Simulation of Cherenkov and diffraction radiations upon periodic metal dielectric structures (review), Procs. Sumy State University. 10(56):110-130 (in Russian).

23. Vorobyov, G.S., Krivets, A.S., Petrovsky, M.V., Tsvyk, A.I., and Shmatko, A.A., (2003), The Smith-Pursell Effect Amplification of the Electromagnetic Waves in an Open Waveguide with a Metal-Dielectric Layer, Telecommunications and Radio Engineering. 59(10):80-92.

24. Vorobyov, G.S., and Tsvyk, A.I., (2002), Diffraction electronics devices with spatially developed structures (review), Procs. Sumy State University. 5(38):158171 (in Russian).

25. Pochanina, I.Ye., (1990), On classification of eigen oscillation modes in open waveguide resonators, Electrodynamics of open structures in $\mathrm{mm}$ - and submm-wave bands. Trans. of papers. Kharkiv: IRE NAS of Ukraine, pp.109-119 (in Russian).

26. Balaklytsky, I.M., Skrynnyk, B.K., and Tretyakov, O.A., (1969), Millimeter and submillimeter wave bands diffraction radiation oscillator, UPJ. 14(4):539-552 (in Russian).

27. Tsvyk, A.I., (1985), Electron-wave theory of diffraction radiation, Trans. AS of UkrSSR. Series A. 12:53-58 (in Russian).

28. Shmatko, A.A., and Odarenko, Ye.N., (2003), Microwave frequencies electronics. Fakt, Kharkiv: 248 p. (in Russian).

29. Cert. of Authorship 593589 USSR, MKI H01J 25/00. Orotron. I.M. Balaklytsky, G.S. Vorobyov, A.I. Tsvyk, Discoveries. Inventions, 1981, 33:327 (in Russian).

30. Marshall, E.M., Philips, P.M., and Walsh, J.E., (1988), Planar orotron experiments in millimeter wavelength band, IEEE Transactions on Plasma Science. 16(2):199205.

31. Ginzburg, N.S., Zavolsky, N.A., and Zapevalov, V.Ye., (2000), Non-stationary processes in orotron with diffraction output of oscillation, JTP. 70(4):99-104 (in Russian).

32. Bratman, V.L., Dumesl, B.S., and Fedotov, A.E., (2002), Broadband orotron operation at millimeter and sub-millimeter waves, International Journal of Infrared and Millimeter Waves. 23(11):1595-1601.

33. Rusin, F.S., Bratman, V.L., and Fedotov, A.E., (2002), Orotron: perspectives of advancing into submillimeter wavelength band, Vacuum microwave electronics: Trans. reviews, pp.121-124 (in Russian).

34. Belous, O.I., Fisun, A.I., and Sukhoruchko, O.N., (2003), Synthesis of Basic Components of a Low-Noise Input Circuit for Millimeter Wavelengths, Telecommunication and Radio Engineering. 59(1-2):111-118. 
35. Belous, O.I., Fisun, A.I., Olkhovsky, I.P., and Sirenko, S.P., (2000), One circuit stabilization of millimeter-wave solid state oscillator by quasi-optical resonator, IJIR\&MMW, 9(9):1451-1462.

36. Peskov, N.Yu., Ginzburg, N.S., Kaminsky, A.A., (1999), High-efficient narrowband maser on free electrons based on Bragg resonator with phase leap, Letters to JTP. 25(11):19-28 (in Russian).

37. Ginzburg, N.S., Rosental, R.M., and Peskov, N.Yu., (2001), Modeling of planar FEL-amplifier with ribbon relativistic electron beam, JTP. 71(12):58-61.

38. Cert. of Authorship 749278 USSR, MKI H01J 25/00. Diffraction radiation oscillator, I.M. Balaklytsky, G.S. Vorobyov, A.I. Tsvyk et al. Discoveries. Inventions. 1982, 35:306 (in Russian).

39. Vorobyov, G.S., Ruban, A.I., and Tsvyk, A.I., (1998), Simulation of excitation mechanisms of oscillation in diffraction electronics devices on coupled open resonators, International Symposium Physics and Engineering of Millimeter and Submillimeter Waves. Kharkov, pp.194-195.

40. Belousov, Ye.V., Korzh, V.G., Koshparyonok, V.N., and Maystrenko Yu.V., (1997), Coupled resonators in dielectrometry of thin films, Radio physics and electronics. 2(1):39-42.

41. Nesterenko, A.V., Tsvyk, A.I., and Shectopalov V.P., (1984), Miniaturization of the diffraction radiation oscillator, Trans. AS of USSR. 277(1):84-88 (in Russian).

42. Androsov, V.P., Vertiy, A.A., Kuzmichev, I.K. et al., (1988), Investigation of gain processes in regenerative amplifier on the basis of DRO, Electronics of millimeterand submillimeter-wave bands, pp.13-17 (in Russian).

43. Vorobyov, G.S., Kryvets, A.S., Petrovsky, M.V., and Ruban, A.I., (2002), Experimental modeling of wave processes in amplifier on Smith-Pursell effect, Procs. of Sumy State University. 5(38):117-124 (in Russian).

44. Vorobyov, G.S., Makeyev, O.S., Pushkaryov, K.A., and Tsvyk. A.I.. (1996), Simulation of transformation of surface waves of the electron beam into volume waves upon metal-dielectric electrodynamic structures, Procs. Sumy State University. 2(6)28-32 (in Russian).

45. Vorobyov, G.S., Pushkaryov, K.A., Ruban, A.I., and Tsvyk, A.I., (1998), Simulation of the excitation processes of diffraction Cherenkov radiation by the electron beam space charge waves on metal-dielectric periodic structures, Third International Symposium Physics and Engineering of Millimeter and Submillimeter Waves. Kharkov, pp.196-197.

46. Vorobyov, G.S., Petrovsky, M.V., and Kryvets, A.S., (2006), On application of quasi-optical open resonant metal-dielectric structures in EHF electronics, Procs. of Higher School. Radio Electronics. 49(7):56-61.

47. Vorobyov, G.S., Tzvyk, A.I., Pushkaryov, K.A., and Makeyev, O.S., (1996), Scattering of electron stream waves on metal-dielectric periodic structures, International Journal of Infrared and Millimeter Waves.17(10):1761-1768.

48. Vorobyov, G.S., Pushkaryov, K.A., and Tsvyk, A.I., (1997), Numerical analysis of screening properties of diffraction grating at excitation with the electron oscillation beam upon metal-dielectric structures, Radioteknika i elektronika. 42:738-740 (in Russian).

49. Vorobyov, G.S., Pushkaryov, K.A., Ruban, A.I., and Tsvyk, A.I., (1998), Power characteristics of spatial harmonics of Cherenkov-diffraction radiation in periodic 
metal-dielectric structures, Procs. of the 8th International Crimea Conference "Microwave equipment and telecommunication technologies". Sevastopol, pp.210212 (in Russian).

50. Vorobyov, G.S., Pushkaryov, K.A., Ruban, A.I., and Tsvyk, A.I., (1999), Power parameters of Cherenkov-diffraction radiation in periodic metal-dielectric structures, Procs. of Higher School. Radio Electronics.42(10):62-66 (in Russian).

51. Vorobyov, G.S., Kryvets, A.S., Shmatko, A.A., and Khutoryan, E.M., (2002), Excitation of oscillations in amplifier on Smith-Pursell effect with metal-dielectric layer, Procs. Sumy State University.5(38):110-116 (in Russian).

52. Vorobyov, G.S., Kryvets, A.S., Shmatko, A.A., and Petrovsky, M.V., (2002), Wave processes in open waveguide with metal-dielectric layer, 12th International Crimea Conference "Microwave equipment and telecommunication technologies" (CriMiCo'2002). Sevastopol, pp.145-146 (in Russian).

53. Kryvets, O.S., and Petrovsky, M.V., (2004), Electron-wave mechanism of amplification of oscillations in open waveguide with metal-dielectric layer, Procs. Lviv University. 37:123-131 (in Russian).

54. Vorobyov, G.S., Kryvets, A.S., Petrovsky, M.V., and Shmatko, A.A., (2004), Electron-wave processes in amplifier on Smith-Pursell effect with metal-dielectric layer, 14th International Crimea Conference "Microwave equipment and telecommunication technologies" (CriMiCo'2004). Sevastopol, pp.209-210 (in Russian).

55. Vorobyov, G.S., (2000), Wave modeling of Cherenkov and diffraction radiation in spatially limited metal-dielectric structures, Radiotekhnika. 116:12-20 (in Russian).

56. Grigoryev, A.D., (1990), Electrodynamics and microwave equipment. Higher School, Moscow: 335 p. (in Russian).

57. Weinstein, L.A., (1988), Electromagnetic waves. Radio i svyaz, Moscow: 440 p. (in Russian).

58. Tretyakova, S.S., Tretyakov, O.A., and Shestopalov, V.P., (1972), Diffraction of wave beams upon plane periodic structrures, Radiotekhnika $i$ elektronika. 17(7):1366-1373 (in Russian).

59. Vorobyov, G.S., Ruban, A.I., and Petrovsky, M.V., (2003), Experimental modeling of conditions of excitation of oscillations in open resonator with metal dielectric structure, 13th International Crimea Conference "Microwave equipment and telecommunication technologies" (CriMiCo'2003). Sevastopol, pp.286-287 (in Russian).

60. Vorobyov, G.S., Petrovsky, M.V., Kryvets, A.S., and Tsvyk, A.I., (2004), On opportunity of application of periodic metal-dielectric structures in EHF electronics, 14th International Crimea Conference "Microwave equipment and telecommunication technologies" (CriMiCo'2004). Sevastopol, pp.211-212 (in Russian).

61. Vorobyov, G.S., Petrovsky, M.V., and Tsvyk, A.I., (2004), Features of spectral characteristics of open resonance electrodynamic systems with periodic metaldielectric structures, The Fifth International Kharkov Symposium on Physics and Engineering of Microwaves, Millimeter, and SubMillimeter Waves. Kharkov, pp.576-578.

62. Vorobyov, G.S., Petrovsky, M.V., Zhurba, V.O., and Krutko, Yu.A., (2005), Perspectives of application of spatially developed resonant structures in diffraction 
electronics, 15th International Crimea Conference "Microwave equipment and telecommunication technologies" (CriMiCo'2005). Sevastopol, pp.265-266 (in Russian).

63. Vorobyov, G.S., Nesterenko, A.V., Pushkaryov, K.A., Tsvyk, A.I., (1992), Investigation of opportunity of increasing efficiency of interaction between electrons and microwave fields in O-type resonant devices, Present-day problems of application physics, pp.101-117 (in Russian).

64. Fisun, A.I., Tkachenko, V.I., Belous, O.I., and Kirilenko, A.A., (2000), Excitation of oscillations in open resonators with echelette and corner-echelette mirrors, Radiotekhnika i elektronika. 45(5):632-639 (in Russian).

65. Fisun, A.I., (1997), Millimeter wave band open resonators with additional restriction of resonant space, IJIR\&MMW. 18(12):2353-2367.

66. Fisun, A.I., (1998), Method analysis of open resonators with step-like deformation of mirrors, Electronic Letters. 4(2):192-193.

67. Weinstein, L.A., (1966), Open resonators and open waveguides. Sov. Radio, Moscow (in Russian).

68. Fisun, A.I., (1997), High-Q resonances in open resonators with partially limited domain, NAS of Ukraine. 12:97-102 (in Russian).

69. Belous, O.I., Fisun, A.I., Kirilenko, A.A., Korneyenkov, V.K., and Miroschnichenko, V.S., (1997), Research on orotron oscillator with dispersive open resonant system, IJIR\&MMW. 18(2):445-461.

70. Petrushin, A.A., Balaklitsky, I.M., and Shectopalov, V.P., (1970), Device for investigation of electromagnetic fields in millimeter-wave band open resonators, PTE. 2:147-151 (in Russian).

71. Vorobyov, G.S., (2000), Substantiation of selection of simulation mode for Cherenkov and diffraction oscillations upon metal-dielectric structures, Procs. of Sumy State University. 16:60-64 (in Russian).

72. Cullen, A.L., (1983), Millimeter-wave open resonator techniques, Int. Journal of Infrared and Millimeter Waves. 10:233-281.

73. Malyshev, V.N., (ed.) (2000), Electrodynamical modeling using the method of finite differences in time domain (FDTD), Saint Petersburg State Electric Engineering University "LEEI" Publishers. 76 p. (in Russian).

74. Churyumov, G.I., Maksimov, I.S., and Yeremeev, D.B., (2003), Mathematical modeling of electromagnetic phenomena using the method of finite differences. 1. General, Radiotekhnika. 135:7-14 (in Russian).

75. Sirenko, Yu.K., (2003), Simulation and analysis of transition processes in open periodic systems, waveguides and compact resonators, EDENA, Kharkov: 363 p. (in Russian). 